\title{
Using Prediction Markets to Track Information Flows: Evidence from Google
}

\author{
Bo Cowgill ${ }^{1}$, Justin Wolfers ${ }^{2}$, and Eric Zitzewitz ${ }^{3}$ \\ 1 Google, Inc, Mountain View, CA, USA \\ bcowgill@google.com \\ 2 Wharton School, University of Pennsylvania, Philadelphia, PA, USA \\ jwolfers@wharton . upenn. edu \\ 3 Dartmouth College, Hannover, NH, USA \\ eric.zitzewitz@dartmouth.edu
}

\begin{abstract}
Since 2005, Google has conducted the largest corporate experiment with prediction markets we are aware of. In this paper, we illustrate how markets can be used to study how an organization processes information. We show that market participants are not typical of Google's workforce, and that market participation and success is skewed towards Google's engineering and quantitatively oriented employees.

We document a number of biases in Google's markets, most notably an optimistic bias. Newly hired employees are on the optimistic side of these markets, and optimistic biases are significantly more pronounced on days when Google stock is appreciating. We also document a reverse favorite longshot bias, where rare events are underpriced by the market. Lastly, we find a bias against "extreme" events: In markets about a continuous variable, traders underprice the high and low ends of the spectrum and overprice the middle.

In the final section of our paper, we document correlated trading among employees who sit within a few feet of one another and employees with social or work relationships. These findings are interesting in light of recent research on the role of optimism in entrepreneurial firms, as well as recent work on the importance of geographic and social proximity in explaining information flows in firms and markets.

The current draft of the full paper can be read at http://bocowgill. com/GooglePredictionMarketPaper.pdf
\end{abstract}

Keywords: prediction markets, information transmission, bias, market efficiancy, peer effects. 